\title{
Adaptive power link adaptation on DVB-T system based on picture quality feedback
}

Tubagus Maulana Kusuma, Randy Rahmanto, Emy Haryatmi

Universitas Gunadarma, Indonesia

\begin{tabular}{l}
\hline \hline Article Info \\
\hline Article history: \\
Received Mar 14, 2018 \\
Revised Apr 1, 2019 \\
Accepted Apr 12, 2019 \\
\hline
\end{tabular}

\section{Keywords:}

DVB

Feedback channel

Link adaptation

Power control

Quality metric

\begin{abstract}
In digital television systems such as DVB-T, service provider has difficulties to observe the quality of picture reception in the viewers' television. This is due to the unavailability of quality feedback sent from viewers' devices to the service provider. Therefore, this research proposes link adaptation method in DVB-T system based on image quality measurement at recipient side, so that service provider may adjust the transmission power in real-time to improve the image quality. Quality metric used in this research is human perception- based no-reference image quality metric, which does not need the presence of the reference frame. The quality assessment is focused on the severeness of blocking artifact, which is the dominant artifacts in MPEG video. The numerical results have shown that power adaptation could maintain good picture quality as well as transmission power efficiency at the same time on the digital television transmission system. The proposed scheme is also suitable for other DVB system as well as various digital television system standards.
\end{abstract}

Copyright (C) 2019 Institute of Advanced Engineering and Science. All rights reserved.

\section{Corresponding Author:}

Tubagus Maulana Kusuma,

Universitas Gunadarma,

Margonda Raya Street, No. 100, Depok 16424, Indonesia.

Email: mkusuma@staff.gunadarma.ac.id

\section{INTRODUCTION}

The advancement in multimedia system triggers the development of the digital television system. A number of standards have been proposed, such as Digital Video Broadcasting (DVB), Digital Multimedia Broadcasting (DMB), Integrated Service Digital Broadcasting (ISDB), and Advanced Television Systems Committee (ATSC) [1-2]. Although there are many available standards, most of the countries around the world adopted DVB system for their national television system standard. DVB is a suite of internationally accepted open standards for digital television. DVB standards are maintained by the DVB Project, an international industry consortium with more than 270 members, and are published by a Joint Technical Committee (JTC) of the European Telecommunications Standards Institute (ETSI), European Committee for Electrotechnical Standardization (CENELEC) and European Broadcasting Union (EBU). DVB system consists of a number of approaches in delivering the broadcast content, such as DVB-T/T2 (terrestrial), DVB-S/S2 (satellite), DVB-C/C2 (cable), DVB-H/SH (handheld). DVB-T was selected in this research due to the free-to-air scheme and it is still widely used by many operators around the world, although DVB-T2 has been introduced.

The DVB-T system uses MPEG-2 Transport Stream (MPEG-2 TS) as the input stream. This stream is transmitted over the channel through DVB-T system. During transmission, the stream might be affected by channel impairments, such as noise, which may decrease the video quality that received by the end user. This problem must be able to be detected and identified by the DVB-T system in order to maintain the picture quality in a good condition. One of the solutions in combating such situation is to increase power. However, increasing power without monitoring channel condition might lead to power inefficiency. 
As the channel condition change over time, thus power adaptation is required in order to maintain power efficiency. Adaptive power control is part of link adaptation strategy, particularly explicit link adaptation. Link adaptation techniques have gained increasing attention for improving the quality of wireless multimedia communication schemes [3-13]. In explicit link adaptation, transmission parameters such as the signal power, the modulation constellation, or the amount of redundancy provided for error control are explicitly adjusted to compensate for the variations in channel conditions. For these purposes, channel state information (CSI) needs to be estimated at the receiver and then communicated back to the transmitter. This feedback enables the transmitter to adapt transmission parameters on a regular basis in response to the CSI at the time. In this research, adaptive power control was selected since it has lower complexity than adaptive modulation and adaptive coding. Adapting the power of the transmit signal in order to account for signal fades that are caused by multipath propagation or other channel impairments, results in the channel being used more efficiently. This can be achieved by controlling the power in such a way that it takes advantage of favorable channel conditions [8-12]. The overall objective of power control is to conserve as much transmit power as possible while at the same time maintaining satisfactory link quality for a wide range of channel conditions. The advantages of power control include reduced interference problems and longer battery life with respect to adapting to small variations in the channel quality. Although power control schemes can be classified in many different ways, the focus of this research is on metrics that are used to initiate the power control algorithm.

This paper is organized as follows. In Section 2, research methods, which contain research approach and design, are discussed. Section 3 presents experiment setup, results from the experiments, and analysis of the results. Finally, concluding remarks and future works are given in Section 4.

\section{RESEARCH METHOD}

In this section, the design of power adaptive DVB-T system that includes DVB-T system block and the adaptive power control block will be discussed. The main DVB-T system that was used during the simulation process using Simulink software is shown in Figure 1.

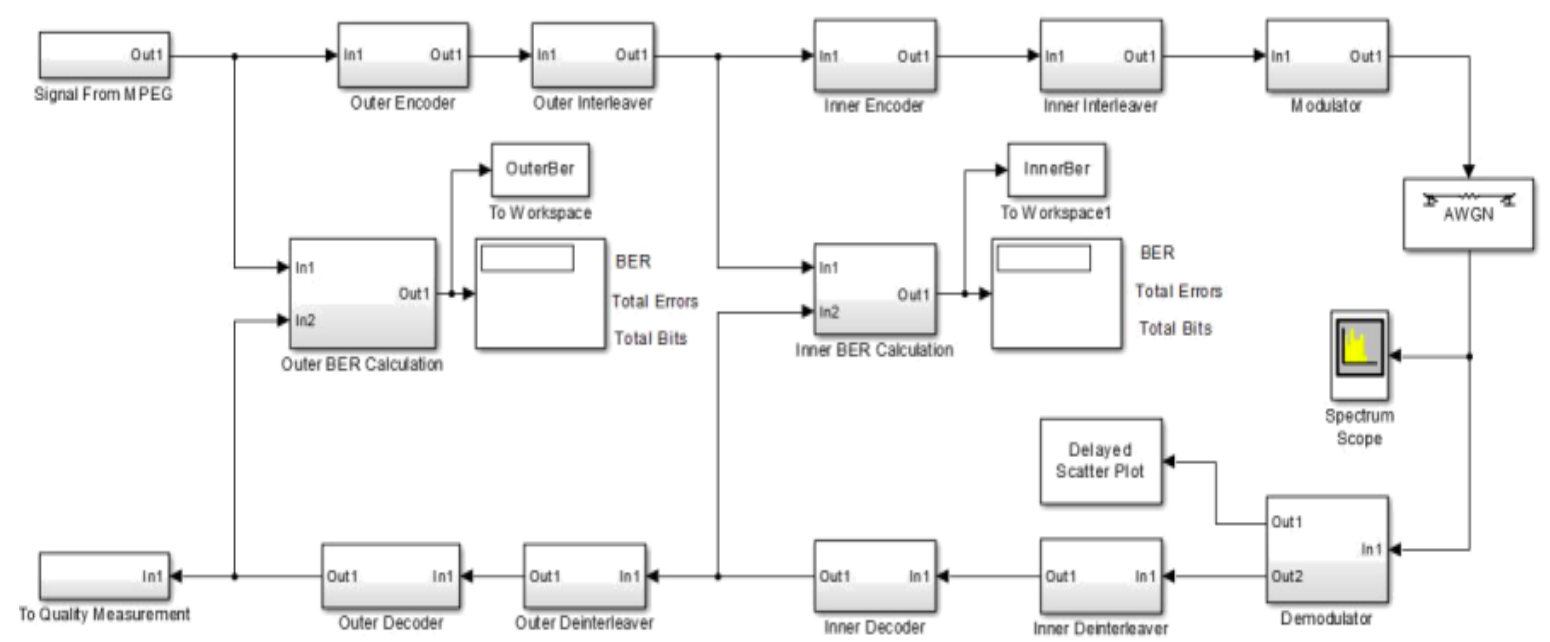

Figure 1. DVB-T system block diagram in Simulink

As shown in Figure 1, DVB-T transmission system takes the input from file in MPEG2-TS or MPEG2 Transport Stream format. Input data is segmented into frames with the length of 188 bytes per frame. The channel encoders consist of outer and inner encoders. For outer encoders, Reed Solomon (RS) $(255,188)$ code was used. This is the reason behind 188 bytes as an input frame length. For the inner encoder, punctured convolutional code with rate 3/4 was used. Outer and Inner interleave were also used to reduce the impact of burst errors by improving the performance of forward error correction codes. The modulation scheme used in the experiment is 64-QAM with 2K-mode Orthogonal Frequency Division Multiplexing (OFDM) transmitter. Outer and Inner decoders were used in the receiver, where Viterbi decoder was used as the inner decoder. The channel used during the simulation is adaptive white Gaussian noise (AWGN) channel. The system block produced 188 bytes of output frame for every 188 bytes of input frame.

Int J Elec \& Comp Eng, Vol. 9, No. 4, August 2019 : 3121 - 3129 
As shown in Figure 2, the proposed DVB-T adaptive power control method is presented. The method consists of picture quality assessment algorithm, feedback channel, and power adaptation scheme. Many different picture quality assessment methods can be used, such as full-reference (a received image is compared with the original or source picture), reduced-reference (features from the received picture are extracted and compared with corresponding stored features of the original or source picture), and noreference (does not require prior knowledge about the original or source image but performs assessment of the received image/blind assessment) [14-15]. However, for real-time picture quality monitoring, the no-reference (NR) is the most suitable method, since no extra information is needed to be communicated to the receiver, as well as no prior knowledge about the original picture is required [16-17]. For real implementation, the feedback or return channel from the receiver to the transmitter for DVB-T system can be based-on general packet radio service (GPRS) or other suitable methods, since the picture quality data is only a few bits in length [16]. The measurement interval can be predefined, for example every 50 frames or every 100 frames. The quality will be the average quality of 50 frames or 100 frames, which will be depended upon the selected predefined interval. Power adaptation scheme will be based on this quality information. When the quality is below a certain predefined quality threshold, then the power will be increased. The power will be decreased when the quality is greater or equal to the threshold. By adapting the power of the transmitter, the picture quality will be maintained in good quality while at the same time maintaining the power efficiency.

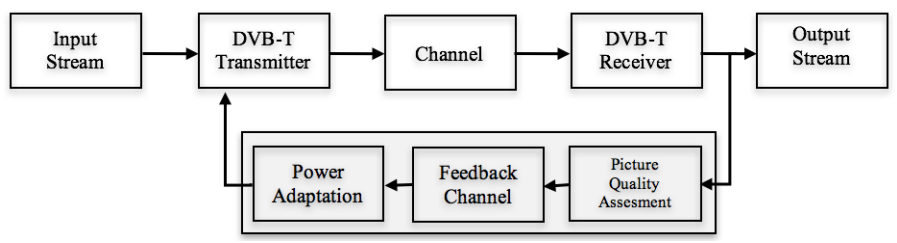

Figure 2. Proposed DVB-T Adaptive Power Control Method

\subsection{Picture quality impairments}

Human vision has the ability to recognize image attributes, such as shape, orientation, size, texture, gradient, luminance, brightness, and contrast. All these attributes contribute to the characteristics of an image or image features, which reveal uniqueness of the image. An undistorted image carries its natural features. However, once these images get corrupted due to compression or transmission, new artificial features, which do not exist in the original images, are introduced. The characteristics of these new artificial features known as artifact characteristics vary from one artifact to another. By knowing the original image features, artificial features introduced by compression errors or transmission errors can be detected. Moreover, the level of severeness of these artifacts can also be measured.

In MPEG video, the impairment is dominated by blocking artifact. Blocking appears in all blockbased compression techniques and is caused by coarse quantization of frequency components. These artifacts can be observed as surface discontinuity or an edge at block boundaries. Non-overlapped block-based scheme, such as DCT, gives rise to the appearance of blocking artifact. The problem with a block-based scheme such as MPEG is that the image is sub-divided into sub-blocks with the size of 8-by-8 pixels each. The transformation and the quantitation processes are then applied to the sub-blocks individually and independently. Correlation among spatially adjacent sub-blocks is not taken into account during the encoding process. As a consequence, smooth transitions between edge boundaries of each sub-block are reduced.

During the decoding process, the edge boundary cannot be fully recovered as it appeared in the original image. The block boundaries are now being visible. Therefore, blockiness or blocking artifact can be easily observed on the image as shown in Figure 3. It is even more noticeable when the bit rate or the number of bits to represent the image is reduced. 

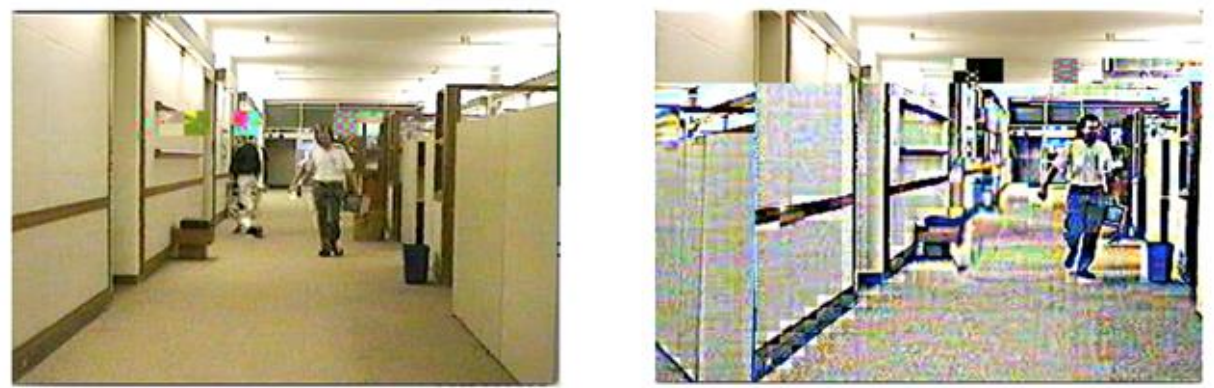

Figure 3. Example of DVB-T picture quality impairments

\subsection{Picture quality metric}

Metric, by definition, is a measure that facilitates the quantification of some particular characteristics. In image processing, metric can be defined as a measurement for assessing the quality of a picture using a quantitative approach. One of the prominent approaches in picture quality measurement is to take into account human visual perception. The picture quality metric that incorporates human visual perception is called perceptual picture quality metric. The measurement is classified into two approaches, which are known as psychophysical approach and engineering approach. Psychophysical approach is based on various aspects of human visual system (HVS) where engineering approach is based on feature extraction of specific artifacts. In this research, engineering approach is used in order to extract specific artifact of MPEG video stream known as blocking artifact. The justification of using engineering approach is the complexity of extracting single artifact is lower than incorporating all aspects of HVS in measuring picture quality, since real-time processing is the main concern in DVB-T picture quality monitoring. The extraction process of blocking artifact will be targeted on the activity on block boundaries. Several techniques, which take human perception into account, have been proposed to extract the information regarding the above artifact, such as proposed by Wang et al. in [14-15].

Wang et al. have proposed a no-reference blocking artifact extraction and measurement model [14-15]. The model is based on the knowledge that blocking artifact has signatures in the spectral domain, which are called the signature of blockiness. They are the peaks in the power spectrum curve of the image at the feature frequencies of $L / 8,2 L / 8,3 L / 8$, and $4 L / 8$, where $L$ is the length of the segment extracted from the signal. The basic idea of this algorithm is to detect the blocky signal and to estimate its power using the assumption that a blocky image is a non-blocky image interfered by an ideal blocky signal. The algorithm starts with calculating the differences between pixels along the row to obtain the horizontal difference, $d_{h}$, and along the column to get the vertical difference, $d_{v}$. Once these differences are calculated, the difference image can be obtained. Next, the blockiness is computed by taking the average differences across block boundaries, $B$. For a $M \times N$ image, the horizontal measurement is defined in [14-15].

$$
\begin{aligned}
& B_{h}=\frac{1}{M(\lfloor N / 8\rfloor-1)} \sum_{i=1}^{M} \sum_{j=1}^{\lfloor N / 8\rfloor-1}\left|d_{h}(i, 8 j)\right| \\
& B_{v}=\frac{1}{N(\lfloor M / 8\rfloor-1)} \sum_{i=1}^{\lfloor M / 8\rfloor-1} \sum_{j=1}^{N}\left|d_{v}(8 i, j)\right|
\end{aligned}
$$

where $(\cdot, \cdot)$ denotes the index of pixel location. In addition to extracting the blocking artifact, this algorithm can also be used to identify the bluriness by observing the reduction of signal activity. It is believed that some insight about the relative blur can be obtained by combining blockiness with the activity measurements [14]. The activity can be measured using two parameters, namely the average absolute difference between in-block image samples, $A$, and the zero-crossing $(\mathrm{ZC})$ rate, $Z$. The first parameter in the horizontal direction can be obtained as [15].

$$
A_{h}=\frac{1}{7}\left[\frac{8}{M(N-1)} \sum_{i=1}^{M} \sum_{j=1}^{N-1}\left|d_{h}(i, j)\right|-B_{h}\right]
$$




$$
\begin{aligned}
& A_{v}=\frac{1}{7}\left[\frac{8}{N(M-1)} \sum_{i=1}^{M-1} \sum_{j=1}^{N}\left|d_{v}(i, j)\right|-B_{v}\right] \\
& Z_{h}=\frac{1}{M(N-2)} \sum_{i=1}^{M} \sum_{j=1}^{N-2} z_{h}(i, j) \\
& Z_{v}=\frac{1}{N(M-2)} \sum_{i=1}^{M-2} \sum_{j=1}^{N} z_{v}(i, j)
\end{aligned}
$$

where

$$
\begin{aligned}
& z_{h}(i, j)= \begin{cases}1 & \text { horizontal ZC at } d_{h}(i, j) \\
0 & \text { otherwise }\end{cases} \\
& z_{v}(i, j)= \begin{cases}1 & \text { vertical ZC at } d_{v}(i, j) \\
0 & \text { otherwise }\end{cases}
\end{aligned}
$$

Then, the mean blockiness, $B$, the mean absolute difference, $A$, and the mean $\mathrm{ZC}$ rate, $Z$, are obtained using the vertical and the horizontal measures.

$$
\begin{aligned}
& B=\left(B_{h}+B_{v}\right) / 2 \\
& A=\left(A_{h}+A_{v}\right) / 2 \\
& Z=\left(Z_{h}+Z_{v}\right) / 2
\end{aligned}
$$

The final blockiness measure, $Q$, can then be obtained using [10],

$$
Q=p 1+p 2 \cdot B^{p 3} A^{p 4} Z^{p 5}
$$

where $p 1, p 2, p 3, p 4$, and $p 5$ denote the prediction parameters that were obtained using curve fitting of the subjective experiment data. The value of $p 1=-245.89, \quad p 2=261.94, \quad p 3=-239.89, \quad p 4=160.17$, and $p 5=64.29$ [10]. The subjective experiment data were obtained from a subjective test, which is complied with ITU-R BT.500 standard [18]. This metric is used for picture quality assessment in this proposed adaptive power control scheme.

\subsection{Adaptive power control}

Power in the wireless system has been part of system resources which needs to be controlled efficiently in order to conserve as much transmit power as possible while at the same time maintaining satisfactory link quality for a wide range of channel conditions. This can be achieved by controlling the power in such a way that it takes advantage of favorable channel conditions [8]. The advantages of power control include longer battery life and reduced interference problems with respect to adapting to small variations in the channel quality. There are mainly four different measurements that can be used to indicate link quality and hence can be deployed with power control algorithms. These are referred to as strengthbased [8], signal-to-interference-ratio (SIR) based [8], BER-based [8] and perceptual quality (PQ) based power control [9-12].

Strength-based power control: The measurement is based on the strength of the signal that arrives at the base station from a mobile terminal. If the signal strength is lower than a given threshold, the power at the mobile terminal will be increased. As the received signal strength increases, the power at the mobile terminal will be decreased. SIR-based power control uses signal-to-interference ratio (SIR) as an indicator. The measurement of link quality exploits signal strength in the presence of channel noise and multi-user interference. If the noise and interference increase, the power control algorithm will be triggered to increase the transmit power. The transmit power will be reduced when noise and interference are decreased. BERbased power control uses bit error rate (BER) as an indicator for the link quality. Similarly, power control may be based on the average number of erroneous frames or FER. Clearly, the transmit power will be increased if the respective error rate exceeds a predefined threshold. Otherwise, the transmit power may be 
reduced accordingly. In a PQ-based scheme, which is based on perceptual quality (PQ), the link quality is captured by a suitable perceptual objective quality metric. Accordingly, transmit power shall be increased when the respective metric indicates a low level of perceptual quality of the received data. The transmit power will be reduced if the quality of the received data is well perceived by the metric.

In this research, PQ-based power control was used. The power was adapted dynamically using ONOFF control scheme. The state diagram of power adaptation process, which is based on picture quality feedback, is illustrated in Figure 4. The picture quality metric used in this scheme is perceptual no-reference blocking metric as proposed in [15]. Blocking metric was used because the dominant artifact in MPEG video is blocking artifact. The process starts by setting the initial power to a predefined value. The video stream is then transmitted using the initial power. At the receiver, the picture is decoded and the perceptual quality (PQ) is measured. The picture quality is in the range of 0 to 100 , where 0 means the worst and 100 means the best [16-18]. Quality threshold (QThreshold) must be set to a predefined value before transmission. In this research, QThreshold was set to 80 , because the picture quality higher than 80 has shown an acceptable quality, so the power will be reduced. If the measured quality is less than or equal to 80 , then the power will be increased. The process is performed until the transmission is terminated.

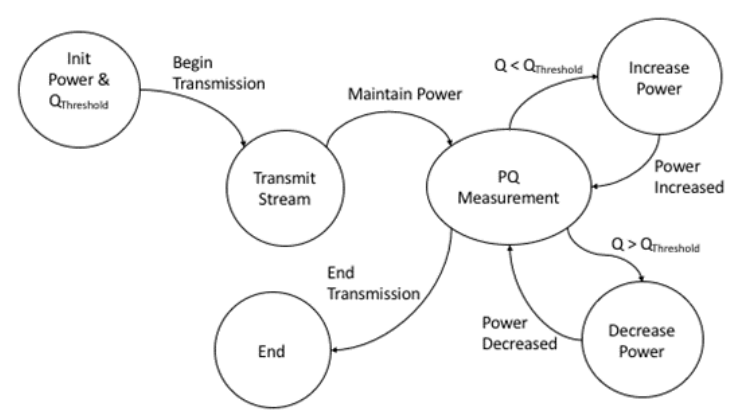

Figure 4. State diagram of PQ-based power adaptation scheme

\section{RESULTS AND ANALYSIS}

In this section, we provide numerical results for test video Hall with the playing duration of 10 seconds, which is encoded in MPEG-2 format with the frame size of $352 \times 240$ pixels. The test video is transmitted through DVB-T system in the Simulink environment, which is based-on the ETSI EN 300744 standard for terrestrial transmission of digital television system [19]. The DVB-T system used throughout the experiments is based-on $2 \mathrm{~K}$ Mode of non-hierarchical transmission [20]. The selected channel for the transmission is adaptive white gaussian noise (AWGN) channel with variable bit energy over noise power spectral density (Eb/No) between $18 \mathrm{~dB}$ to $19 \mathrm{~dB}$. The $18 \mathrm{~dB}$ starting point was selected because the test video could only be viewed on that value. Below $18 \mathrm{~dB}$ the stream was heavily corrupted and could not be opened, thus the perceptual quality could not be measured. As shown in Figure 5(a), the bit error rate (BER) was decreased when the Eb/No was increased.

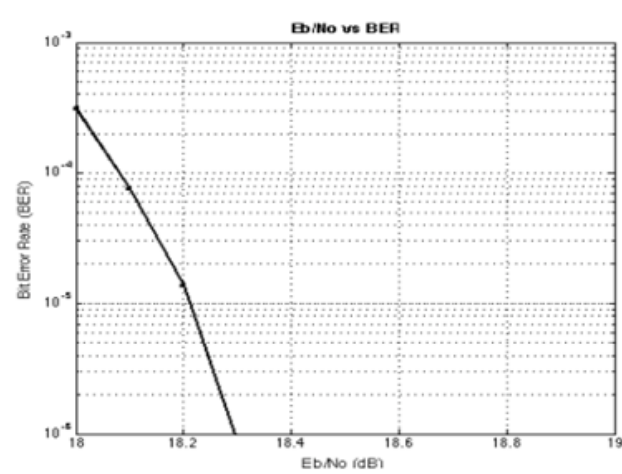

(a)

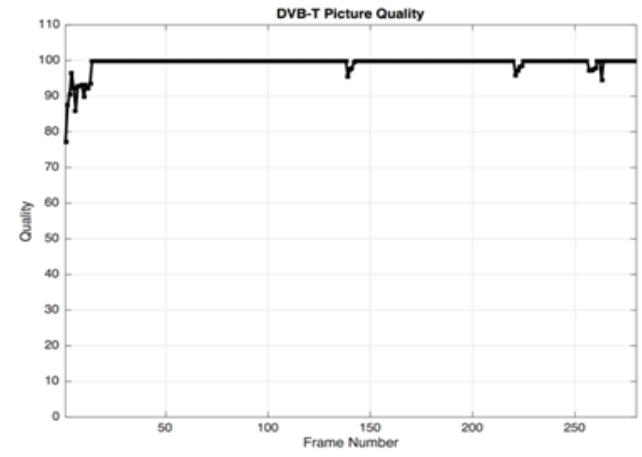

(b)

Figure 5. (a) Eb/No (dB) vs BER and (b) DVB-T objective picture quality 
If video quality is measured using perceptual image quality metric, it can be observed that the quality varies among frames, which was influenced by bit errors during transmissions, as presented in Figure 5(b). The metric range is set between 0 to 100, where 0 means worst quality and 100 means excellent quality. The Eb/No was set to $18 \mathrm{~dB}$.

In utilizing power adaptation, the impact is shown in Figure 6(a). It is clearly demonstrated that the power is fluctuated due to the channel conditions. The power adaptation scheme control the power of the transmitter based on the feedback from the receiver. The information sent from the receiver contains picture quality information which is used by the transmitter to decide whether to increase or to reduce the transmission power. The role of the power adaptation scheme is to keep the power minimum while maintaining acceptable picture quality in the receiver.

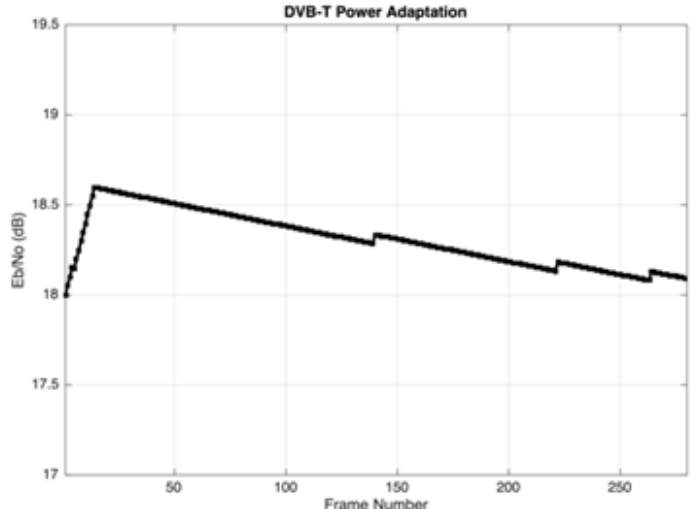

(a)

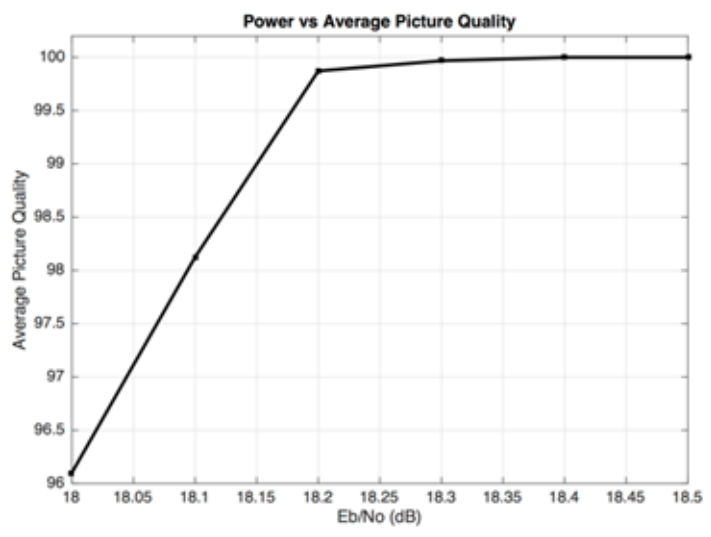

(b)

Figure 6. (a) DVB-T power adaptation and (b) power (dB) vs picture quality

The relation between average picture quality and power is presented in Figure 6(b). It is shown that the average picture quality is increased when the power is increased, which have shown that the power correlates well with picture quality. It reached the average picture quality of 100 (highest quality) when the power is $18.4 \mathrm{~dB}$ and it does not change even when the power is higher than $18.4 \mathrm{~dB}$. As the results of the abovementioned dynamic power adaptation, picture quality improves gradually as shown in Figure 7. When the power is increased, the picture quality is also improved significantly.

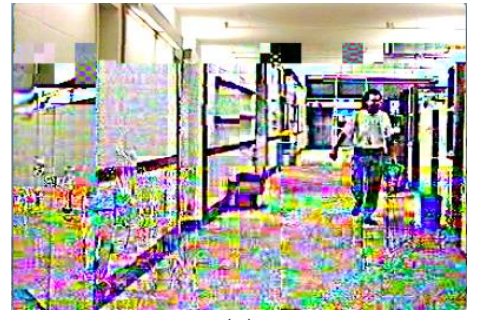

(a)

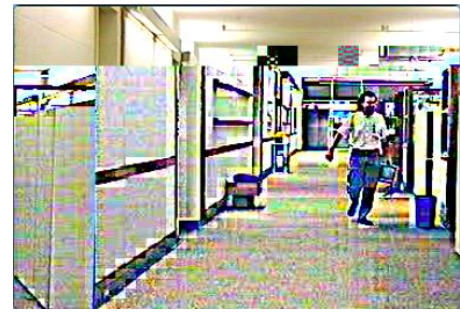

(b)

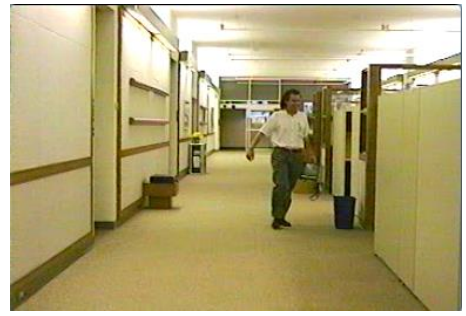

(c)

Figure 7. Example of quality improvement with dynamic power adaptation: (a) $\mathrm{Q}=81$, (b) $\mathrm{Q}=85$, and (c) $\mathrm{Q}=100$

\section{CONCLUSION}

A novel power control scheme for DVB-T system is presented, which takes the user perceived quality into account. The scheme is based on no-reference perceptual picture quality metric, which does not require a reference picture at the receiver and is therefore well suited to real-time applications. The scheme is designed to measure the quality of the received picture and to maintain the transmission power based on the measured picture quality. Feedback/return channel is used to carry channel state information based on perceptual quality measurement. It was shown by way of experiment that the proposed adaptive power control could maintain good picture quality as well as transmission power efficiency at the same time. 
Further investigation is needed to find more sophisticated control algorithm so that the power control scheme may achieve a more effective adaptation to the changes in channel conditions. This will continuously keep the picture in a good quality during the transmission under a predefined quality threshold, while maintaining the efficiency of power utilization. The proposed scheme is also suitable for other DVB system as well as various digital television system standards.

\section{REFERENCES}

[1] W. Fischer, "Digital Video and Audio Broadcasting Technology: A Practical Engineering Guide," Heidelberg: Springer, 2010.

[2] El-Hajjar M, Hanzo L, "A Survey of Digital Television Broadcast Transmission Techniques," IEEE Communications Surveys Tutorials, 15(4), 1924-1949, 2013.

[3] T. M. Kusuma, M. Caldera and H.-J. Zepernick, "Utilising objective perceptual image quality metrics for implicit link adaptation," 2004 International Conference on Image Processing, ICIP '04, Singapore, 2004, pp. 2319-2322, 2004.

[4] C. Morel, P.-D. Arapoglou, M. Angelone, and A. Ginesi, "Link adaptation strategies for next generation satellite video broadcasting: A system approach," vol. 61, pp. 603-614, Dec. 2015.

[5] M. Angelone, A. Ginesi, E. Re, and S. Cioni, "Performance of a combined dynamic rate adaptation and adaptive coding and modulation technique for a DVB-RCS2 system," in Proc. IEEE $6^{\text {th }}$ Advanced Satellite Multimedia Systems Conference and 12 ${ }^{\text {th }}$ Signal Processing for Space Communications Workshop, pp. 124-131, Sep 2012.

[6] S. Andrenacci, D. Spano, D. Christopoulos, S. Chatzinotas, J. Krause, and B. Ottersten, "Optimized link adaptation for DVB-S2X precoded waveforms based on SNIR estimation," in Proc. IEEE 50 ${ }^{\text {th }}$ Asilomar Conference on Signals, Systems and Computers, pp. 502-506, Nov 2016.

[7] G. Ku and J. M. Walsh, "Resource Allocation and Link Adaptation in LTE and LTE Advanced: A Tutorial," in IEEE Communications Surveys \& Tutorials, vol. 17, no. 3, pp. 1605-1633, 2015.

[8] D. M. Novakovic and M. L. Dukic, "Evolution of the power control techniques for DS-CDMA toward 3G wireless communication systems," May 2000. [Online]. Available: http://www.comsoc.org/pubs/surveys

[9] A. Selmi, M. Siala and H. Boujemaa, "Efficient link adaptation using joint power control, adaptive modulation and coding with enhanced ARQ protocol," 2016 International Symposium on Networks, Computers and Communications (ISNCC), Yasmine Hammamet, pp. 1-4, 2016.

[10] B. Rohani and H.-J. Zepernick, "Application of a perceptual speech quality metric for link adaptation in wireless systems," in Proc. IEEE $1^{\text {st }}$ International Symposium on Wireless Communication Systems, pp. 260-264, Sep 2004.

[11] E. Danish, A. Fernando, O. Abdul-Hameed and M. Pourazad, "Perceptual quality-driven resource allocation in energy-aware wireless video multicasting," 10th International Conference on Heterogeneous Networking for Quality, Reliability, Security and Robustness, Rhodes, pp. 101-107, 2014.

[12] S. E. Ghoreishi, A. Aijaz and A. H. Aghvami, "Delay-Constrained Video Transmission: A Power-Efficient Resource Allocation Approach for Guaranteed Perceptual Quality," 2015 IEEE Global Communications Conference (GLOBECOM), San Diego, CA, pp. 1-7, 2015.

[13] E. Danish, A. Fernando, O. Abdul-Hameed, M. Alshamrani and A. Kondoz, "Perceptual QoE based resource allocation for mobile 3D video communications," 2014 IEEE International Conference on Consumer Electronics (ICCE), Las Vegas, NV, pp. 454-455, 2014.

[14] Z. Wang, A. C. Bovik, and B. L. Evans, "Blind measurement of blocking artifacts in images," in Proc. IEEE International Conference on Image Processing, pp. 981-984, Sep 2000.

[15] Z. Wang, H. R. Sheikh, and A. C. Bovik, "No-reference perceptual quality assessment of JPEG compressed images," in Proc. IEEE International Conference on Image Processing, pp. 477-480, Sep 2002.

[16] T. M. Kusuma and H. J. Zepernick, "In-service image monitoring using perceptual objective quality metrics," Journal of Electrical Engineering, vol. 54, pp. 237-243, Dec 2003.

[17] U. Engelke, T. M. Kusuma and H. Zepernick, "Perceptual Quality Assessment of Wireless Video Applications," 4th International Symposium on Turbo Codes \& Related Topics; 6th International ITG-Conference on Source and Channel Coding, Munich, Germany, pp. 1-6, 2006.

[18] "ITU-R Recommendation BT.500-13: Methodology for the subjective assessment of the quality of television pictures," Geneva, Switzerland, 2012.

[19] "ETSI EN 300744 v1.6.2.: Digital Video Broadcasting (DVB); Framing structure, channel coding and modulation for digital terrestrial television," European Broadcasting Union (EBU), Cedex, France, 2015.

[20] N. A. Kotak, B. S. Sedani and V. V. Dwivedi, "Simulation \& Performance Analysis of DVB-T System Using Efficient Wireless Channels," 2011 International Conference on Computational Intelligence and Communication Networks, Gwalior, pp. 677-681, 2011. 


\section{BIOGRAPHIES OF AUTHORS}
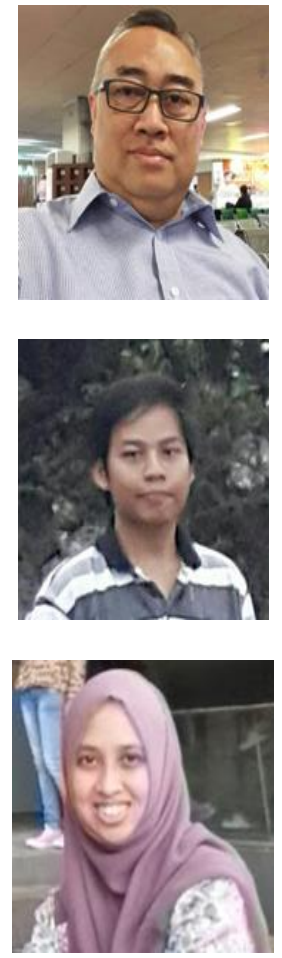

Tubagus Maulana Kusuma received B.Eng. degree from Universitas Gunadarma, Jakarta, Indonesia, in 1994. He continued his study at Curtin University of Technology, Perth, Western Australia and received MEngSc. and PhD degree in 1999 and 2006, respectively. Dr. Kusuma holds the position of the Director of Graduate Masters Program in Technology and Engineering Universitas Gunadarma, Jakarta, Indonesia. Dr. Kusuma is a member of IEEE and has served as a reviewer of several scientific journals. His research interests include image and video processing, multimedia communications, digital broadcasting, and perceptual multimedia quality assessment.

Randy Rahmanto received B.Eng. degree from Universitas Gunadarma, Jakarta, Indonesia, in 2015. He continued his study at the same university and received MEng. degree in 2016. Mr. Rahmanto is currently working as a professional software developer. His research interests include software engineering and telecommunications.

Emy Haryatmi received B.Eng. degree from Universitas Gunadarma, Jakarta, Indonesia, in 2000. She continued her study at Curtin University of Technology, Perth, Western Australia and received MEngSc in Telecommunications and Networking in 2004. In 2016, she obtained her $\mathrm{PhD}$ degree from Universitas Gunadarma. Dr. Haryatmi holds the position of Program Secretary of Master's Program in Electrical Engineering Universitas Gunadarma, Jakarta, Indonesia. Her research interests include computer networks and digital broadcasting. 